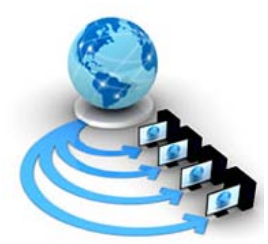

Volume 10, No. 3, May-June 2019

\title{
THE PROGRAMMING LANGUAGES: A SURVEY STUDY
}

\author{
Ruchi Sharma \\ Research Scholar ,Department of Computer Science, \\ Himachal Pradesh University, Shimla, India
}

\begin{abstract}
Since the beginning of computers, thousands of programming languages are designed. However, a comparatively few proportions of those really placed to sensible use within the trade. Some languages are developed and/or adopted by the academic community for teaching functions. Three of the most widespread programming languages C, C\# and Java are chosen to be the objects to study. A Survey was conducted; feedback from students also gathered to evaluate which language is better in the following parameters: Readability, Reusability, Portability, and Expressiveness.
\end{abstract}

Keywords: C, C\#, Java, Survey, Readability, Reusability, Portability, Expressiveness.

\section{INTRODUCTION}

Programming languages are notations. They are used for specifying, organizing and reasoning concerning computations. Even as English compositions vary from notes to sonnets; programs vary from prototypes that are used once and forgotten to production tools that are shared and supported. This vary of wants has intended the creation of many programming languages.

A programing language ought to even be fairly natural for determination issues, a minimum of issues among its supposed application space. For example, a programming language whose only data types are numbers and arrays may be natural for determination numerical issues, however would be less natural for determination issues in commerce or artificial intelligence.. Conversely, a programming language whose only data types are strings associated lists would be an unnatural selection for determination numerical issues [1].

The development of general purpose digital computers with keep programs was initiated within the early 1950s. Early programs were written directly in computer code. This presently gave thanks to assembly languages. Assembly languages square measure extremely machine dependent and typically referred as low-level languages. Programming language design and implementation strategies have evolved unendingly since the earliest high level languages appeared within the 1950s [2].

\section{The C Programming Language}

C is a programming language developed at AT\& T's Bell Laboratories of USA in 1972. It turned into designed and written through a person named Dennis Ritchie. Within the late $70 \mathrm{~s} \mathrm{C}$ commenced to update the greater familiar languages of that time like PL/I, ALGOL, and many others. $\mathrm{C}$ turns into very famous because it is dependable, easy and smooth to apply. Furthermore, in an enterprise where more modern languages, tools and technologies emerge and vanish day in and day out, a language that has survived for more than three a long time has to be absolutely desirable[3].

\section{The C\# Programming Language}

C\# changed into created at Microsoft late within the 1990s and turned into part of Microsoft's overall .NET strategy. It became first launched in its alpha version within the middle of 2000. C\#'s chief architect changed into Anders Hejlsberg. The grandfather of C\# is C. From C, C\# derives its syntax, a lot of its key phrases, and its operators. C\# is a new computer programming language advanced by means of Microsoft corporation, USA. C\# is a totally objectorientated language and is the first component-oriented language. It has designed to support the important thing capabilities of .NET framework. It is easy, efficient, productive and type-secure language derived from the popular c programming language. Even though it belongs to the family of $\mathrm{C}$, it's far merely object-oriented, modern language suitable for growing web-primarily based packages. C\# is designed for building robust, dependable and durable additives to handle real world packages [4].

\section{The JAVA Programming Language}

Java turned into conceived by way of James Gosling, Patrick Naughton, Chris Warth, Ed Frank, and Mike Sheridan at solar Microsystems, Inc. in 1991. It took 18 months to develop the primary operating model. This language changed into first of all known as “Oak," but was renamed "Java" in 1995. Object-oriented programming (OOP) is at the core of Java. In reality, all Java programs are to at least a few quantity object-oriented. All computer programs encompass two factors: code and data. Moreover, a program may be conceptually organized round its code or round its data. This is, a few applications are written around "what is taking place" and others are written round "who is being affected." These are the two paradigms that govern how a software is built. The primary way is called the process-orientated model. This method characterizes a application as a series of linear steps (this is, code). The process-oriented model can be idea of as code performing on data. To control growing complexity, the second one method, referred to as object-oriented programming, was conceived. Object-orientated programming organizes a program round its facts (that is, objects) and a set of 
properly-described interfaces to that data. An objectorientated program can be characterized as information controlling get right of entry to code [5].

\section{RESEARCH METHODOLOGY}

The descriptive approach is also used for obtaining the results. A survey has conducted manually among IT and Computer Science students. In all cases, printed or verbal explanatory notes were provided to respondents to ensure consistent interpretation of the terminologies and questions in the questionnaire. Out of 105 questionnaires distributed, 100 are valid. On the basis of the Questionnaires, analysis has been done.

\section{QUESTIONNAIRE FORMAT}

The questionnaire has two parts. The first part contained basic information of the respondent(Name, Name of the Organization, Course enrolled and Semester). The second part consisted 15 questions. Each question was based on the parameters which we used for the study.

\section{RESULTS AND ANALYSIS}

The items of the questionnaire were analyzed and the results are discussed below with the help of graphs.

Language Better in terms of Readability: The language help or hinder good programming practice. A language that enforces cryptic syntax, very short identifiers, default declarations, and an absence of type information makes it difficult to write readable code. The significant point is that code is read (by its author and other programmers) more often than it is written.

It is evident from figure 1 , that $16 \%$ students report says $\mathrm{C}$ while $14 \%$ C\#, 52\% Java and rest $18 \%$ all of the languages are better in terms of readability.

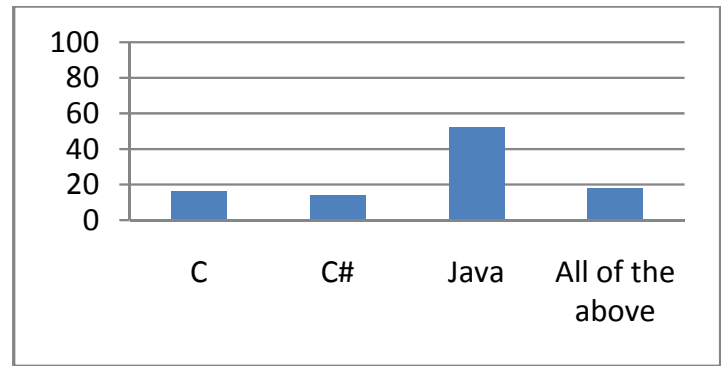

Figure 1: Better in Readability

Preferred Language: From the analysis, it is found that 34\% students prefer C language, 7\% C\# and 59\% Java, depicted in Figure 2.

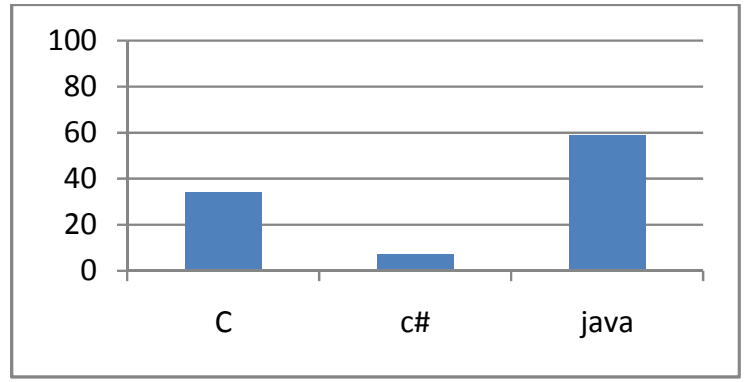

Figure 2: Preferred Language

Programming Language Supports Effective Reusability: The language support effective reuse of program units. If so, the project can be accelerated by reusing tried-and-tested program units; it might also develop new program units suitable for future reuse. Relevant concepts here are packages, abstract types, classes, and particularly generic units.

As depicted in Figure 3, 13\% students says C language supports reusability, $15 \% \mathrm{C \#}$ and $71 \%$ says Java and $1 \%$ says no language supports reusability.

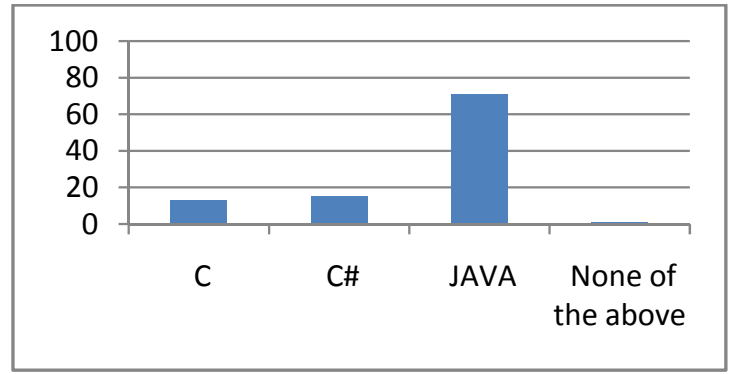

Figure 3: Programming Language supports effective reusability

Language which is Platform Independent: The languages help or hinder writing of portable code. In other words, the code can be moved from one platform to a dissimilar platform without major changes. From the analysis, it is found that $8 \%$ students prefer C language, $7 \%$ C\#, 68\% Java and $17 \%$ prefer all languages for platform independency, depicted in Figure 4.

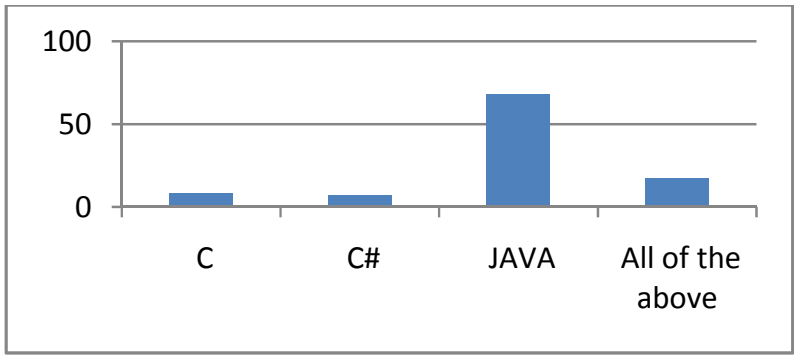

Figure 4: Platform Independency

Programming Language Better in terms of Expressiveness: This factor reflects the ability of a language to express complex computations or complex data structures in appealing, intuitive ways In terms of expressiveness, 19\% students prefer C, 14\% C\#, 65\% Java and $2 \%$ students says no language is better in expressiveness as shown in Figure 5. 
Ruchi Sharma, International Journal of Advanced Research in Computer Science, 10 (3), May-June 2019,89-91

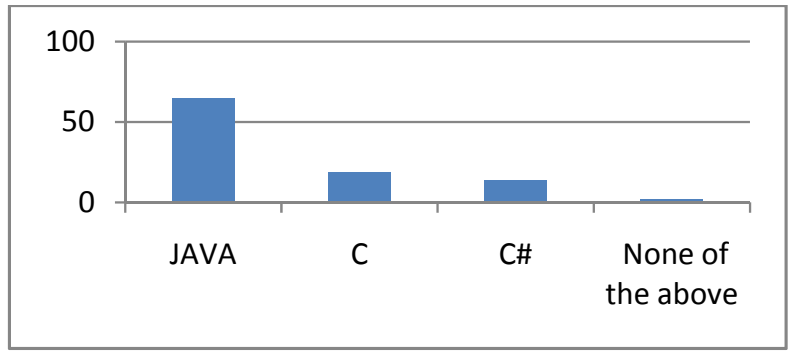

Figure 5: Better in terms of Expressiveness

\section{CONCLUSION}

The analysis and discussion leads to the conclusion that Java language is frequently used by students. In terms of readability java becomes first and second language is c. Programming language which supports effective reusability is java and second language is c\#. For platform independency again java becomes first. In terms of expressiveness java becomes first and c becomes second programming language.The results obtained from survey are represented. From the analysis, it has also found that java is best for all parameters.

\section{REFERENCES}

[1] Lutz Prechelt, "An Empirical Comparison of Seven Programming Languages”, IEEE, pp. 23-29, 2000.

[2] Terrence W. Pratt and Marvin V. Zelkowitz, Programming Languages Design and Implementation, Fourth Edition, Prentice Hall, Inc, New Delhi , 2001.

[3] Yashavant P. Kanetkar, Let Us C, Tenth Edition, BPB Publication, New Delhi, 2010.

[4] Herbert Schildt, The Complete Reference C\# 4.0, Tata McGraw-Hill, New York, 2010.

[5] Herbert Schildt, The Complete Reference Java , Seventh Edition, Tata McGraw-Hill, New Delhi, 2007.

[6] Ranjit Singh, Research Methodology, Third Edition, Sage Publications, New Delhi, 2011.

[7] Hieu Vu, "FROM C TO C++, C\#, AND JAVA", International Journal of Scientific Research and Innovative Technology,Vol.2, pp.80-84,Feb.2015. 